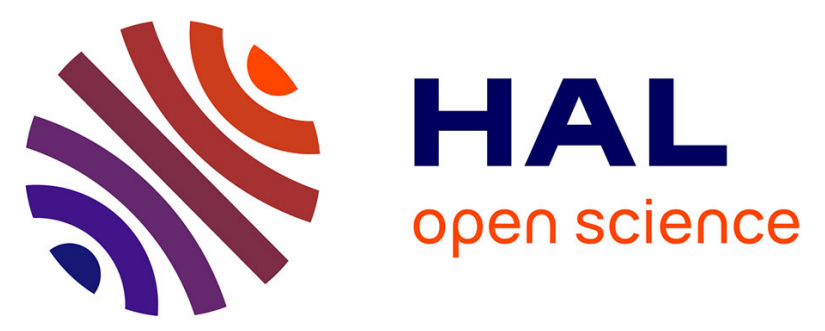

\title{
Du papier au numérique: étude exploratoire des usages des collections de presse ancienne et des pratiques afférentes
}

\author{
Céline Paganelli, Stéphanie Pouchot, Evelyne Mounier
}

\section{To cite this version:}

Céline Paganelli, Stéphanie Pouchot, Evelyne Mounier. Du papier au numérique: étude exploratoire des usages des collections de presse ancienne et des pratiques afférentes. "Les intersections: gens, lieux, information", 40ème congrès annuel de l'Association Canadienne des Sciences de l'Information, Université du Nouveau-Brunswick et Université Saint-Thomas, Fredericton, Nouveau-Brunswick, Canada., Jun 2011, Fredericton, Nouveau-Brunswick., Canada. p.28. hal-00651683

\section{HAL Id: hal-00651683 https://hal.science/hal-00651683}

Submitted on 14 Dec 2011

HAL is a multi-disciplinary open access archive for the deposit and dissemination of scientific research documents, whether they are published or not. The documents may come from teaching and research institutions in France or abroad, or from public or private research centers.
L'archive ouverte pluridisciplinaire HAL, est destinée au dépôt et à la diffusion de documents scientifiques de niveau recherche, publiés ou non, émanant des établissements d'enseignement et de recherche français ou étrangers, des laboratoires publics ou privés. 
Céline Paganelli

Université Montpellier 3, France ; Laboratoire Gresec, Université Grenoble 3

Evelyne Mounier

Université Grenoble 2, France ; Laboratoire Gresec, Université Grenoble 3

Stéphanie Pouchot

Université de Lyon, France ; Université Claude Bernard Lyon 1, ELICO, EA 4147

\section{Du papier au numérique : étude exploratoire des usages des collections de presse ancienne et des pratiques afférentes}

Résumé : Notre recherche vise à mieux cerner et comparer les usages des collections de presse ancienne papier et numérisée. Elle s'appuie sur les collections de journaux du XIX ${ }^{\mathrm{e}}$ siècle de la bibliothèque municipale de Lyon et appréhende les pratiques de ces lecteurs en matière de recherche d'information et de consultation.

Abstract: The goal of our research is to better identify and understand the use of old print collections in paper and electronic formats. Work is based on newspaper collections from the $19^{\text {th }}$ century held at the Lyon public library and reports on readers' information-seeking and consultation practices.

\section{Contexte général}

La presse ancienne représente, notamment pour les chercheurs, les étudiants et les journalistes, une source de connaissances considérable. Elle permet de s'informer sur la vie politique, économique, sociale et culturelle d'une époque, l'ancrage local de certains titres présentant, lui, un creuset d'informations sur l'histoire d'une région. Mais les collections de presse sont fragiles et leur manipulation délicate. Ainsi, leur numérisation vise-t-elle un double objectif : la valorisation de ce patrimoine documentaire et la conservation des originaux. La bibliothèque municipale de Lyon (France) s'inscrit dans cette dynamique en numérisant depuis 2007 les collections de la presse illustrée rhône-alpine du XIX $\mathrm{X}^{\mathrm{e}}$ siècle.

Notre étude prend place au sein d'un projet visant à valoriser et mettre en ligne ces collections patrimoniales. L'un des volets de ce projet a pour objectif d'appréhender les attentes et usages en matière de consultation des collections de presse ancienne (papier et numérisée). Ici, l'analyse des attentes et des pratiques des usagers est entendue comme un préalable à une réflexion et à des préconisations sur les traitements documentaires et sur les modalités d'accès aux documents numérisés.

L'accès aux collections papier et le travail sur ces documents sont communs dans toutes les salles de lecture et les usagers habituels sont relativement bien connus. Les modes d'accès en ligne posent en revanche davantage de questions : Quels objectifs peuvent amener un usager à utiliser les collections de presse ancienne? Quels types de contenus le lecteur vient-il chercher et sous quelle(s) forme(s) (texte, gravure...) ? Comment cherche-t-il ? Est-il indifférent de tourner les pages d'un journal ou de consulter à l'écran? Est-ce plus simple de formuler quelques mots de 
requête ? Quels usagers nouveaux peuvent se manifester? Quelles sont les pratiques émergentes liées au passage au numérique?

Les pouvoirs publics français affichent, certes, une volonté de s'intéresser à la manière dont ces fonds numérisés sont utilisés (Lesquins, 2006), ainsi l'appel à projets $2010 \mathrm{du}$ Ministère de la Culture concernant la numérisation du patrimoine culturel mentionne « une attention particulière [ ...] à l'émergence d'outils et de services favorisant des usages culturels innovants par les internautes ». De même, les professionnels impliqués dans ces projets expriment leur intérêt pour connaître les motivations et les comportements de leurs usagers. Pourtant, peu d'études portent sur les usages effectifs des collections numérisées.

\section{Hypothèses}

Nous envisageons les hypothèses suivantes :

- Les besoins et attentes de l'usager habituel des fonds de presse ancienne peuvent ne pas différer selon le support.

- Toutefois, la mise en ligne des collections induit une diversification et un élargissement des publics potentiels. Ainsi, de nouveaux besoins et attentes peuvent émerger qui ne sont pas nécessairement pris en compte par les interfaces de recherche et de consultation.

- Enfin, la structure bien particulière des documents de presse induit des habitudes d'exploration et des formes d'utilisation spécifiques. Il se peut que la lecture à l'écran et/ou l'interface de recherche entraînent des pratiques ou stratégies différentes.

\section{Collecte et analyse des données}

\section{Une enquête par questionnaires}

Nous avons choisi de recueillir les données selon deux modalités : via un questionnaire sur internet, ciblant les lecteurs du Progrès Illustré numérisé et mis en ligne, et via un questionnaire papier, destiné aux usagers se déplaçant à la bibliothèque de la Part Dieu (l'un des sites de la BM Lyon), sur rendez-vous, pour consulter des titres de presse ancienne. Ils ont été distribués à ces usagers à leur arrivée à la banque d'accueil. Les questionnaires en ligne et papier comportaient respectivement 27 et 26 questions permettant, entre autres, de cerner les motivations des répondants, leurs stratégies de recherche et de consultation ainsi que leur niveau de satisfaction par rapport aux informations trouvées.

Le nombre de questionnaires colligés est peu élevé : seules 40 réponses au total ont été recueillies ${ }^{1}$. Toutefois, les résultats de nos deux enquêtes concordent avec les tendances mises en évidence lors des études menées sur les publics de Gallica (Lupovici, 2003) et Europeana (Lesquins, 2007).

Aperçu des résultats 
En premier lieu, notre hypothèse quant à l'élargissement des publics est vérifiée. En effet, lorsque ce type de collections est numérisé, ce ne sont plus seulement des chercheurs ou spécialistes qui les consultent ; le grand public est également intéressé, que ce soit par curiosité ou pour des recherches plus ciblées, en lien avec son histoire familiale ou des événements survenus dans sa région (quand il s'agit de consulter la presse locale). Par ailleurs, ce sont la plupart du temps des personnes ne fréquentant pas la bibliothèque en tant que lieu physique. Cet élargissement est également géographique : comme pour l'étude Gallica, les publics consultent les collections à partir de différentes régions françaises ou de l'étranger.

Notre étude suggère par ailleurs que le cadre dans lequel la consultation des collections est effectuée influence les stratégies mises en œuvre. Aussi les motivations et objectifs principaux des usagers influencent-elles les pratiques informationnelles qui en découlent. D'autres études l'ont déjà montré, le contexte professionnel et les contraintes spécifiques de la tâche ont un impact sur l'activité d'information (Jarvelin, 2004). Plus largement, la recherche d'information apparaît comme un processus affecté par le contexte et l'activité à mener (Bartlett, 2005), (Guyot, 2002).

Le recours à la presse ancienne dans un cadre professionnel ou universitaire cible la recherche d'informations factuelles: l'usager met alors en œuvre des stratégies spécifiques, fonction du degré de précision de l'information recherchée. Ainsi, il peut parfois expliciter de manière idoine ce qu'il veut trouver, tant au niveau du contenu informationnel que de sa forme espérée : tel lieu, tel événement, tel personnage, des gravures, des cartes, etc. Dans d'autres cas, à l'inverse, il ne dispose que de pistes ou d'indices trop flous pour pouvoir formuler clairement son besoin. C'est le cas de la notion d'«événement» qui revient régulièrement parmi les objectifs exprimés, sans qu'elle soit précisément définie.

Cette catégorie d'usagers a l'habitude de dépouiller la presse, de lire les documents au moins en diagonale, appuyant sa lecture sur des points de repères directement liés à la structure propre de chaque journal : la « une » ou des rubriques spécialisées. Concurremment, les consultations dans un cadre personnel favorisent le butinage, la "promenade » dans les collections, même si, là aussi, il peut y avoir besoin d'accéder à une information donnée.

La mise en ligne élargissant les accès, la proportion des consultants relevant du grand public est accrue. Ceci explique probablement l'importance des visiteurs du Progrès Illustré version web qui, par ailleurs, ne sont pas des habitués des bibliothèques. De plus, la récence de la mise en ligne a probablement augmenté le nombre d'internautes arrivant par curiosité ou par hasard sur la base en ligne de ce titre et qui ne recherchaient rien en particulier (peut-être même découvraientils tout juste l'existence de l'outil).

S'agissant des types d'informations recherchées par les internautes, il existe une différence entre les lecteurs habitués à la consultation des exemplaires papier des journaux, notamment pour des raisons professionnelles, et les autres. Pour la première catégorie, le media ne semble pas influencer les types d'informations recherchées. En revanche, pour la seconde catégorie, il est intéressant de noter la place qu'occupe le document iconographique, que l'usager recherche le plus souvent au hasard, en parcourant les pages, en trouvant ou non. Sans doute pourrait-on là imaginer des fonctionnalités de recherche spécifiques au niveau des interfaces. 
En outre, conformément à l'une de nos hypothèses, les répondants n'ont pas la même stratégie de consultation selon qu'ils sont face à la presse ancienne sur son support original et sur support numérique en ligne. Cette observation est particulièrement vraie pour les internautes déjà habitués des bibliothèques et qui recourent aux journaux anciens dans le cadre d'une activité bien ciblée. Dans ce cas, la stratégie habituelle consiste alors en un dépouillement très fouillé et une lecture souvent approfondie des numéros ou articles sélectionnés. En ligne, l'usager ne peut dépouiller la presse de manière aussi précise soit parce que les outils ne le permettent pas, soit parce que, sur une longue durée, la lecture à l'écran est jugée inconfortable.

\section{Pour conclure : des objectifs et stratégies multiples à prendre en compte}

Les résultats de cette étude exploratoire ne sont certes pas généralisables. Néanmoins, ils confirment que les objectifs des usagers consultant la presse ancienne sont variés et que les stratégies mises en œuvre le sont tout autant. Il est donc essentiel que les interfaces de consultation prennent en compte cette hétérogénéité des besoins et des pratiques. Cela peut se traduire aussi par la constitution de parcours thématiques permettant de guider les usagers grand public et de satisfaire leur curiosité (Clavier, 2010) le répérage des " événements 》 objectif de recherche d'un panel large d'usagers, La mise en contexte du corpus (Cazenave, 2004) devrait également contribuer à améliorer les interfaces, de même que le développement d'outils favorisant une lecture confortable pour les usagers spécialistes mettant en œuvre un dépouillement précis et systématique. Par exemple, la modélisation du rubriquage permettrait aux usagers habituels de retrouver un cadre connu et de faciliter leur recherche ou la lecture en diagonale.

\section{Références bibliographiques}

Bartlett, Joan, et Elaine Toms. 2005. How is Information Used? Applying task analysis to understanding information use, Actes de la conference ACSI/CAIS, Londres.

Cazenave, Jean, Pantxika Dagorret, Christophe Marquesuzaa et Ga Mauro. 2004. La revitalisation numérique du patrimoine littéraire territorialisé. Colloque "Le numérique : impact sur le cycle de vie du document", organisé par l'EBSI et l'ENSSIB, Montréal, 13-15 octobre 2004. [en ligne] consulté le 10/01/2011 http://www.enssib.fr/bibliotheque-numerique/document-1213

Clavier, Viviane. 2010. Indexer des parcours thématiques pour valoriser les collections de presse numérisée, CIDE, 13ème congrès international sur le document électronique, Paris, 16-17 décembre 2010.

Guyot, Brigitte. 2002. Une activité de travail méconnue : l'activité d'information. Colloque ICUST usages.

Jarvelin, Kalervo et Peter Ingwersen. 2004. Information seeking research needs extension towards tasks and technology. Information Research, 101 paper 212. [en ligne] <http://InformationR.net/ir/10-1/paper212.html> Consulté le 14 janvier 2011 
Lesquins, Noémie et Valérie Tesnière. 2006. La bibliothèque numérique européenne, $B B F, n^{\circ}$, p. 68-80 [en ligne] <http://bbf.enssib.fr/consulter/bbf-2006-03-0068-012> Consulté le 14 janvier 2011

Lesquins, Noémie. 2007. Europeana : rapport de bilan sur les usages et attentes des utilisateurs, Bibliothèque nationale de France, direction des Services et des réseaux, département de la Bibliothèque numérique [en ligne] <http://www.bnf.fr/documents/europeana_2007.pdf $>$ Consulté le 14 janvier 2011

Lupovici, Catherine, Thierry Cloarec et France Charentenay. 2003. Les usages de Gallica. BBF, 2003, $\mathrm{n}^{\circ} 4$, p. 40-44 [en ligne] <http://bbf.enssib.fr/consulter/bbf-2003-04-0040-007> Consulté le 14 janvier 2011 